\title{
A Three-Tier Framework for Understanding Disruption Trajectories for Blockchain in the Electricity Industry
}

\author{
Almero de Villiers and Paul Cuffe, Member, IEEE
}

\begin{abstract}
Ever since the invention of Bitcoin by the pseudonymous Satashi Nakamoto, cryptocurrency has provoked debate in banking and finance sectors, and is sometimes considered a potential successor to fiat currency. Blockchain, the new technology underpinning decentralised and immutable databases, has seen much discussion as a potentially game-changing development. Although many industries are exploring its value, the technology has thus far made only minor impacts. A rapidly expanding base of research has emerged on blockchain's role as a potential disruptor in the electrical energy industry. However, it may be difficult to distinguish hype from more imminently plausible impacts. This paper attempts to serve as a guide for engineering management wishing to make sense of blockchain's potential in electricity. This is accomplished by formulating a novel blockchain industry disruption framework, which exists across three tiers. These tiers extend from ideas with the least effect on an industry to total revolutionary concepts that could completely transform an industry. This taxonomy is constructed by examining existing research into disruption hierarchies and blockchain classification methods. Through the lens of this taxonomy, a literature review is performed on blockchain's role in energy to draw out themes and ideas characterising each tier. The potential likelihood of real-world application of various ideas are discussed, giving consideration to how established industries may be affected or disrupted. The authors provide some conjecture here. Finally, courses of action are suggested for those whose sector may be affected by blockchain.
\end{abstract}

\section{Managerial Relevance Statement}

Engineering management personnel in the electricity industry may be interested in exploring the

A. de Villiers (almero.devilliers@ucdconnect.ie) and P. Cuffe (paul.cuffe@ucd.ie) are with the School of Electrical and Electronic Engineering, University College Dublin.

This publication has been funded by the Sustainable Energy Authority of Ireland under the SEAI Research, Development \& Demonstration Funding Programme 2018, grant number 18/RDD/373. potential blockchain holds as a tool, both in current and future climates. However, blockchain has been the subject of much hype and, as such, there may be confusion around it's true practicality. This study is conducted as a guide for the perplexed engineering manager i.e. as a manual for making decisions in application of the emerging blockchain technology in their specific sector. Existing and hypothetical examples are examined so as to give tangible demonstrations of implemented ideas for management. Thus, a roadmap of industry change is formulated and sectors that seem most susceptible to disruption are examined, and advice is given for future-proofing.

\section{INTRODUCTION}

I INCE Satashi Nakamoto's seminal whitepaper $\checkmark$ proposing the cryptocurrency Bitcoin was published in 2008 [1], blockchain has gained much notoriety [2]. The technology is most often associated with cryptocurrency, largely due to the latter's highprofile agitation of banking and finance. Cryptocurrency has become a hot topic in investment circles, as more and more individuals acquire cryptographic assets [3], such as Ether, Litecoin or the aforementioned Bitcoin. As increasingly numerous outlets and services begin accepting cryptocurrencies payment, it has become difficult to ignore its current and potential impact as an alternative to fiat currency.

But what is blockchain? The term refers to a ledger of data which is decentralised, immutable and can be monitored with no trust from any party involved [2]. Blocks of stored transactions are strung together using hash chains [4]. When a new transaction is completed, it is automatically transmitted to all parties, who then have the option to recognise it (and thus add it to the accepted canonical 
ledger) or reject the contribution. The commonly used proof of work mechanism has miners solving complex mathematical problems. These problems are asymmetrical in difficulty i.e. they are difficult to solve but easy to verify [2]. Thus, the system will add blocks of transactions as they are successfully verified [4]. The miner who performed the work of solving these problems is granted cryptocurrency as a reward for securing the network. An alternative consensus method is proof of stake. This method, while still largely experimental, does not rely on computing power. Rather it makes use of the idea of ownership within the network [4], using a game theory system. Users who own more of the cryptocurrency or who have a better reputation thus have more voting power. In terms of applications of these methods, Bitcoin and most other blockchains, use the proof-of-work system.

The next major evolution in the blockchain space came in the form of smart contracts. These were first conceptually proposed by Nick Szabo in 1997 [5] as immutable scripts that execute automatically under specific circumstances, without any third party involvement [6]. The first, and most notable platform for actually implanting smart contracts comes in the form of Ethereum, a next-generation blockchain [7] that functions as a Turing-complete world computer [8]. Smart contracts on the Ethereum blockchain process cryptographic assets strictly according to predefined rules. The aptness of the execution of these smart contracts is verified using similar principles to how Bitcoin transactions are verified. For this reason, the execution of a smart contract cannot be impeded or interfered with by any party.

Blockchain now has potential beyond merely being a decentralised database, potentially acting as a tool for new business systems, models and applications. Smart contracts allow cryptographic wealth to be transferred and handled according to prestated conditions, creating a paralegal space where "code is law". They also potentially present a new and exotic method of mediating relationships between people, organisations and governments. Therefore, any trust-based industry may be seriously affected by an uptake in their use. Smart contracts may take over finance handling from banks [9]. The services of notaries and lawyers could be largely automated [10], with blockchain-based trust and regulation mechanisms coming to the forefront. While this may all be conceptually true, there has been very little implementation in reality. None of the many Ethereum-based dapps (decentralised apps) have seen significant uptake, and may still be deemed novelties. For instance, the MakerDAO, the most popular Ethereum dapp at the time of writing has just over 2400 users [11], and can thus hardly be considered mainstream.

Various sectors are considering adopting blockchain in some form or another [12], [13]. This interest is likely due to various unique features, including its security, stability and decentralised nature [13]. For instance, the online gambling industry has, to some extent, embraced the technology [14] [15], using cryptocurrency as a payment layer, as well as making use of the secure ledgers for information exchange. The insurance sector has also explored blockchain usage as a secure public store for client assets [16], while the real estate industry is considering storing land titles in a similar manner [17]. The field of logistics and supply chain management is undergoing a more notable transformation due to its adoption of blockchain [18], largely as a tracking database. The technology allows for simplified transparent tracking by using a public blockchain as a ledger. Blockchain is also being used as a tool for tracking ownership of copyright and other intellectual property [19].

Recent times have seen also an increase in research proposing blockchain as a potential tool for the energy industry [20], [21]. Proposed energy related blockchain-based ideas range from the straightforward, such as crytpocurrency rewards for renewable generation [22] and local energy trading [23], to the more exotic, such as federated power plants [24] and restructured finance mechanisms for renewable energy projects [25]. Due to an enthusiasm around the concept, and with much media attention, blockchain has, however, perhaps become a "buzzword" within the technology sector [12], [26]. Thus, there may be a confusion and hesitance concerning its value in the electricity industry. Furthermore, it is unclear when and how the rising tide of blockchain will affect 
the electricity industry, or if it will at all. Many concepts are either in a purely conceptual stage or have only been embraced in a limited manner. All this uncertainty may lead to confusion for engineering management who are seeking to use the technology to add value to their specific sector.

The use of smart contracts to mediate relationships in the electricity sector has also been suggested, such as in [27], where they are used to perform price negotiation and automatic settlement and payment after use. As this paper attempts to examine, the traditional centralised structure of the energy industry (in terms of ownership, physical structure and governance), with limited interaction and data-flow, could potentially be upturned by the introduction of blockchain and smart contracts (at least according to some exuberant commentary). Involved parties may all be given a voice in a future energy supply structure [28]. This could result in a new form of shared governance, spreading responsibility across all involved. That is to say, important decisions regarding, for instance, grid expansion, operation and generation technology make-up, could be made collectively by all energy consumers and producers. In a similar vein, finance for large-scale projects may make use of smart contract technology [25]. This will enable the general energy consuming public to become stakeholders in large-scale generation. Thus, blockchain technology may have the potential to disrupt the purchase and supply of electricity at all points in the value chain. If and how these exotic concepts are to gain public acceptance remains to be seen.

This paper attempts to serve as a guide for management personnel and strategic decision makers wishing to make sense of blockchain's role in the energy sector, as well as those who are understandably sceptical. In Section II a novel taxonomy of blockchain is formulated, ranging from superficial impacts to complete industry disruption. This exists across three tiers, the defining characteristics of which are expanded on in Section II. The taxonomy attempts to describe the general steps of disruption of an industry by blockchain. Blockchain technology's use in the energy industry specifically is then examined in a general literature review in Section III through this lens. Existing and hypothetical applications of blockchain technology are sorted by the three tiers. The Disruptive Technology tier includes some conjecture by the authors, such as a fully blockchain-based energy supply system that has not been discussed in depth thus far in the existing literature. The authors also provide some thoughts on proposed ideas and their future trajectories, including real world suitability and the likelihood of mainstream uptake. The section concludes with a speculative summary of the most likely outcomes for blockchain's role in the energy industry.These ideas are examined through the lens of the above-mentioned novel taxonomy.

\section{Methodology}

This section describes a novel methodology for conducting a literature review of the extant proposals for blockchain use in the electricity industry. The guiding principle for this literature review is the classification of ideas according to the depth of disruption they portend for the industry. This is to provide industry management and decision makers with a "funnel" of potential future industry trajectories. Three identified tiers make up a blockchain taxonomy consisting of a spectrum from sustaining technology to deep potential disruption of the established sector. These tiers are formulated so as to evaluate the potential effect an idea may have on its relevant industry, thus serving as a guide for the literature review. They are informed by the range of blockchain philosophies and conceptualisations, from benign database to radical liberating technology.

\section{A. Taxonomy Formulation}

When examining the exact process of how a technology disrupts an industry, literature can be traced back as far as Diffusion of Innovations, a book first published in 1962 [29]. In it, author Everett Rogers theorises about the spread and adoption of an innovative idea. Rogers' theory puts emphasis on four elements that affect the uptake of an idea, namely the validity of idea itself, the means of communicating the idea, passage of time and the prevailing social systems [29]. These are streamlined into five stages [29]:

- Knowledge. 
- Persuasion.

- Decision.

- Implementation.

- Confirmation.

Rogers' system served as partial inspiration to the Four Stages of Disruption formulated by Steven Sinofsky of venture capital firm Andreessen Horowitz (notable for their sizeable investments in blockchain concepts, such as Coinbase, Ripple and OpenBazaar, amongst others). However, Sinofsky argues that Rogers' stages of diffusion struggle to fully characterise disruptive technology [30], something perhaps difficult to predict in the 1960s.

"A central question to disruption is whether it is inevitable or preventable. History would tend toward inevitable, but an engineer's optimism might describe the disruption that a new technology can bring more as a problem to be solved."

- Steven Sinofsky, Andreessen Horowitz [30]

Sinofsky's four tiers of disruption are as follows [30]:

- Disruption of incumbent.

- Rapid linear evolution.

- Appealing convergence.

- Total re-imagining.

These tiers are loosely based on Elisabeth KüblerrRoss' classical five stages of grieving (denial, anger, bargaining, depression and acceptance) [31], taken from the viewpoint of the incumbent i.e. the "disruptee".

Richard Adler, of international think tank Aspen Institute authored a piece with another view on technology disruption in [32]. A chapter in this report titled The Taxonomy of Disruption proposes two broad categories, the first of which is disrupting the scale of operations [32]. This category claims that disruption can occur by either increasing fragmentation of an industry, or by increasing concentration of an industry. The second type of classification is disrupting ways of connecting with others [32]. This is split into two concepts again, namely redefining relationships and accelerating learning.

The novel taxonomy proposed in this paper attempts to focus on blockchain technology's very specific path of disruption, so the concepts above by Rogers and Sinofsky are perhaps too broad. The Aspen Institute's method closes in on the intended target, but is again too general. Kilkki et. al construct a more specific layered framework for disruption and the interactions involved [33]. A brief summary of their complex model is as below [33]:

New theory $\rightarrow$ New technology $\rightarrow$ Technology acquires patents $\rightarrow$ Technology results in value generation model $\rightarrow$ Industry architecture changes $\rightarrow$ Consumer behavioural changes.

The eventual outcome has implications for society and authorities, even resulting in new laws and regulations [33].

Closing in on blockchain and smart contracts as specific subject matter, Wang et. al hypothesise on future smart contract developments in three steps as below [34]:

- Formal verification.

- Layer two.

- Smart contract driven parallel organisation and societal management.

The first category focuses on applying proof that a smart contract can fulfil its formal specification. The second category covers the rise of a smart contract based ecosystem acting as a consensus mechanism for the transition of states and token payment. Wang et. al's final category is smart contract driven parallel organisation and societal management i.e. governance mechanisms [34]. Similarly, Lu et. al, in their study on blockchain's use in the oil and gas industry, divide up the use of the technology into three main categories [35] ${ }^{1}$ :

- Trading.

- Management/decision making.

- Supervision.

On a slightly different tangent, Bryan Smith of Coin Insider arranges blockchain technology into three generations [7].

- Generation 1: Cryptocurrency, store of value, transfer of value between peers without a central authority.

\footnotetext{
${ }^{1} \mathrm{Lu}$ et. al include another category focusing on cybersecurity, which
} is not relevant to the present study. 
- Generation 2: Smart contracts and associated mechanisms, management of digital assets (inspired largely by the Ethereum project).

- Generation 3: Future developments.

In the formulation of this paper's taxonomy, the authors settled on a three tier structure, as opposed to the sources above which use four, five or more categories. The first tier attempts to condense some of the ideas above, such as Sinofsky's disruption of incumbent and Adler's scale of operations disruption. Kilkki et. al's steps, up to and including their value generation model, are considered here. The final, and most pervasive idea is taken from Smith's first generation of blockchain. The first tier is thus established as using blockchain for commodities and transactions. This tier is discussed in Section II-B1. This level of blockchain penetration is likely to have no more than minor effects on industry, and can be likened to natural technological advance i.e. sustaining technologies.

The intermediate tier of disruption is equated to Smith's second generation blockchain. The first three stages of Sinofsky's disruption model are condensed here, with communication being the primary quality. Killki et. al's industry architecture is relevant to some degree, but perhaps not on a major scale. Lu et. al's first two categories are considered, as trading and management mechanisms are the primary focus of smart contract usage thus far. Adler's second category sub-classification of redefining relationships is also applicable. All these ideas considered, the second proposed tier considers derivatives and ecosystems i.e. smart contract ecosystems with associated mechanisms for trading, communication, management etc. Smart contracts act as an automated treasurer in asset handling. This is expanded on in Section II-B2. Wang et. al's idea of societal management/governance mechanisms are perhaps the most accurate summary of the proposed second tier. This tier could result in significant disruption for an industry, but not cause a total shift in structure and operation.

The third tier of disruption tends towards more exotic concepts. This is inspired by Sinofsky's final category of complete re-imagining, as well as Lu et. al's third blockchain category of supervision methods. Kilkki et. al describe the logical final conclusion as implications for society and authorities. The primary focus of the third tier of the proposed blockchain taxonomy is thus established as ownership structures and governance, as expanded on in II-B3. This level of industry disruption by a blockchain is likely to have severe impacts on an industry, thus the designation as Disruptive Technology.

\section{B. Taxonomy Structure}

This section expands on each of the tiers of blockchain disruption, exploring the typical attributes and examples within each category. The tiers are summarised in Table I. This table includes the defining characteristics of each of the tiers, and attempts to visually demonstrate their operational structures.

1) Tier 1: Sustaining Technology: Subject matter grouped within this tier consists of technologies and ideas that, if adopted, would allow existing industries to continue with their current business model. These technologies are generally complimentary within the current paradigm, and manifest as additional layers to industry working. No governmental permission or regulatory changes are required. A company embracing this technology may have a small team assigned to overseeing implementations and relevant operations.

Thus, tier one requires minor or no physical infrastructure change and largely piggybacks off of existing systems. There is little involvement by utilities or governing bodies. Adding a cryptocurrency payment layer requires only digital and administrative changes. This tier can be compared to to generation 1 blockchain technology, such as Bitcoin, which is mainly focused on the store and transfer of value [7].

2) Tier 2: Evolutionary Technology: Ideas grouped in this tier generally would require some form of restructuring of businesses within an industry. These are concepts that would, to some extent, replace currently accepted mechanisms if adopted. If a company were to utilise this technology, they might require a full department to implement and oversee its development and application.

3) Tier 3: Disruptive Technology: This section examines the deepest tier of disruption. Most concepts within this tier require a significant overhaul 
of industry/sector workings. Emphasis is placed on the potential governance and ownership aspects of blockchain i.e. radical new ways of structuring paralegal relationships between entities. This section for categories based on a what could be case, as there are very few real-world applications thus far i.e. a futurist outlook.

\section{Guided Literature Review}

This section uses the blockchain disruption taxonomy of Section II to conduct a structured literature review of the electricity sector to provide a guide for the perplexed. The taxonomy also allows the authors some conjecture. Concepts based on similar or related ideas are grouped into the various subsections below using the three tiers. Relationships, similarities and differences between these underlying ideas are explored and commented on. Table II groups and summarises the examined concepts by the three tiers of disruption established before. This table includes the defining characteristics of each of the tiers in the electricity sector, and attempts to visually represent their operational structures. Citations for the various sources examined are also grouped by their approximate tier. The table's third tier (Disruptive Technology) also includes citations, but these are for publications on which the more abstract ideas (such as those in Section II-B3) are based.

This section serves as a guided tour through the existing ideas for engineering management and decision makers interested in blockchain as potential tool for their industry. A recent comprehensive literature review on blockchain-related activities and initiatives in the electricity sector has been performed by Andoni et. al [37] and this publication is referred to often in the present paper. The concepts and ideas discussed in their piece are examined through the lens of the taxonomy developed in Section II.

\section{A. Sustaining Technology}

This taxonomic level is proposed to capture ideas that will have minimal effect on existing industries as whole. These are generally concepts that work as complimentary services. No or little additional physical infrastructure is required, such as smart meters, processing equipment, Internet of Things (IoT) enabled devices etc. That is to say, these concepts will usually piggyback on existing infrastructure and industry paradigms, with no direct involvement from the existing regulating bodies.

The primary focus of this tier is on commodities and transactions. Cryptocurrency is the simplest application, serving to facilitate the store and transfer of funds. As a payment layer alone it is likely to have only a minor impact on an industry, especially if existing coins are used, rather than minting an application-specific coins. Advantages are the same as any case where cryptocurrency is used over fiat currency [38]. Distributed ledger technology, however, provides a secure and transparent method of asset management [16], and acts as a secure public database for keeping track of ownership.

Tokenisation is a major theme in this tier. This is the process whereby a physical product or asset is represented cryptographically, and can thus easily be traded, transferred, stored or analysed. The concept is expanded on, and the exact process of energy tokenisation is explained in III-B1.

1) Crytpocurrency as Payment Layer: The simplest blockchain-based application in the energy sector consists of allowing energy purchase with cryptocurrency as payment layer. Established cryptocurrencies have been gaining ground as a new method of electronic payment [39]. Services such as Living Room of Satoshi, Enercity [37] and other gateways make payment of electricity bills with cryptocurrency possible. These businesses usually accept cryptocurrency and pay electricity bills on behalf of clients using fiat currency. However, a number of energy utilities, such as Dutch energy provider BAS [37], have begun accepting Bitcoin directly, without a mediator. Another related application consists of rewarding producers of home generation with special cryptocurrencies. Once a producer has registered for the program, they can verify their production and receive cryptocurrency in return. Verification can be done by manually submitting meter readings [22], or automatically by internet-enabled smart meter if the producer has invested in this. It should be noted that this process may be subject to the Oracle problem [40], as it relies on trustworthy access to off-chain data. Reward can take the form of a preexisting coin such as Bitcoin in the case of [41], or 
TABLE I. General Blockchain Industry DisRuption Classification

Tier

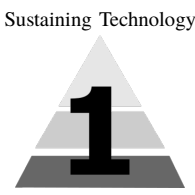

Evolutionary Technology
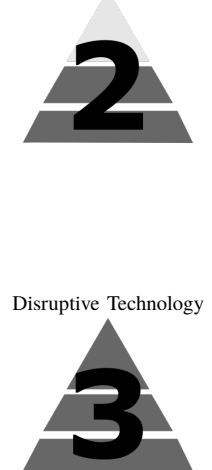

Quotes

Traits \& Uses

"Bitcoin has proven effective as a store of value" [7]

"The value of Bitcoin as store of value, medium of wealth transfer and in future...medium of consumer exchange is indeed based on its strong and seamlessly global security" [36]

"Second-generation blockchains, such as Ethereum threaten not only to disrupt our understanding of a monetary system but could further pose serious consequences for litigators, regulators, and officials."

"We are now seeing innovative approaches that help participants dramatically expand the scope and substance of their relationships with others, opening up stance of their relationships with other
new forms of collaboration..." [32]

“...truly significant disruptions affect...entire industries and even society: former industrial leaders may vanish and be replaced by new entrants...and the new market conditions emerging from the disruption may require significant adaptations at the level of societies in terns of new instintions and re levetion." [33]

"The reimagined technology often has little resemblance to the legacy...The melding of old and new into a completely different solution often creates whole new categories of products and services, built upon a base of technology that appears completely different." [30]
- Smart contract ecosystem.

- Revised and automated handling of relationships between people.

- Decentralised Autonomous Organisations

- Meta-data analytics

- Requires significant additional infrastructure.

- Some regulator involvement.

- Blockchain-based decentralised governance

- Total overhaul of payment and governance structure.

- Largely replaces incumbant industry, becomes the new standard.

- Considerable societal impact.

- Regulatory changes of some kind required.
Typical Structure
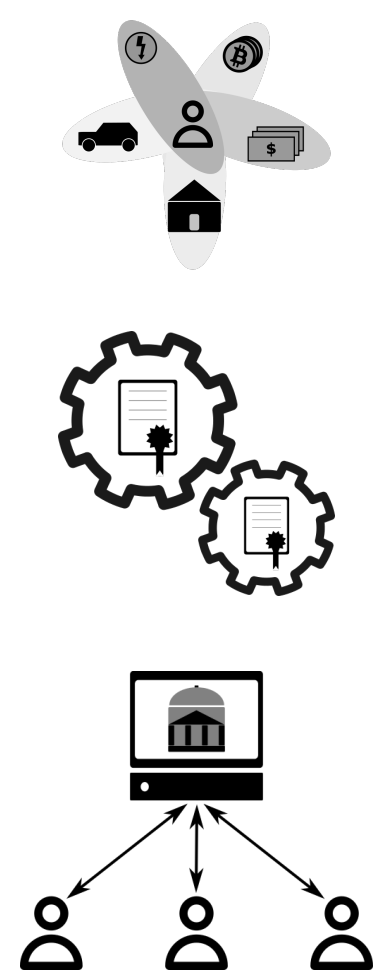

an application-specific coin such as Solarcoin [22]. A potential producer may be incentivised to invest in generation assets (such as home-scale PV or wind) if they could receive cryptocurrency reward for their production. This could be a more attractive option than simply receiving a small credit on their bill from the utility operator when exports are measured. Another related example attaches the value of a cryptocurrency to a weighted aggregate of renewable energy markets [42]. Utility operators could provide reward for assisting with grid stability, such as supplying reactive power, load shifting for demand side management and providing a "clean" waveform with low harmonic content.

For electricity wholesalers, cryptocurrency billing may be a potential method of streamlining the payment process, by switching over to a purely digital system (i.e. without any relationship to fiat currency). It may also present a simple method of rewarding feed-in generation. If the company is unwilling to engage in the admittedly tumultuous sector of traditional cryptocurrency value fluctuation, they may consider adopting an existing stablecoin instead, such as DAI [43], Libra or Digix Gold Tokens.

In these examples no tokenisation of energy takes place (defined in Section III-B1) and a smart meter is not necessarily required. Utilities would only be involved on a minor scale, agreeing to accept cryptocurrency as payment, and metering would be adequate in its existing common "dumb" form. In the authors' opinion, these concepts are likely to become more commonplace in the coming years, but unlikely to create major disruption within the industry as a whole on their own.

2) Blockchain Notarisation: Distributed ledger technology can serve as an immutable digital notarisation; the time [44], date and contents of a transaction cannot be changed once added to the blockchain [10] and is visible to all. Thus, blockchain contents 
cannot be refuted due to the associated timestamp. This has been proposed as tool to keep track of renewable energy credits [45] and carbon credits [46]. Furthermore, it can be used to keep track of ownership of generation equipment and electrical infrastructure, especially in shared ownership schemes, such as those discussed in Section III-C1. Records for Company A's carbon emissions may be stored on a blockchain by a trusted service, making it possible for regulators to track emission performance. It may also allow for simplified carbon credit trading; Company B may purchase some number of A's carbon credits, perhaps with cryptocurrency. In another example, a wind farm company could keep a list of their assets on the blockchain, making ownership records transparent. Fraudulent behaviour could be decreased in this way. The notarisation aspect and its use in the energy sector are primary characteristics of the Sustaining Technology tier, as ownership and commodity tracking are the focus. Blockchain's usage as a notarisation tool seems likely to become commonplace in the coming years.

\section{B. Evolutionary Technology}

This tier serves to categorise ideas that may cause significant electricity industry disruption. The primary focus is on derivatives (as mentioned above), implemented within a smart contract-based ecosystem. Smart contracts allow for new approaches to multi-party, derivative and complex transactions [6]. Residing on the blockchain, they can be accessed by any party, thus making their workings fully transparent [47]. They also allow for some level of autonomous operation and can be used to to disintermediate current operations. Exchanges with users can be handled automatically, with assets being received and distributed according to a predefined set of rules [48]. Smart contracts can thus make for exotic trading arrangements that facilitate the emergence of an ecosystem. Groups of smart contracts can be used to form Decentralised Autonomous Organisations $(D A O s)$. A DAO functions like a virtual mediator, enforcing and executing specific rules and actions [44], accomplished without human management or directors [49]. This allows for a new take on finance, similar to online crowd-sourcing models, such as
Kickstarter or GoFundMe, but without any trusted intermediate service.

Considering the above, some form of infrastructure change is required for ideas within the Evolutionary Technology tier, most likely IoT enabled devices that can communicate with the relevant blockchain. With smart contracts taking on a more prevalent role, there may be some regulatory/legislature change required to accommodate this [50]. This is likely to mean that existing regulators play some role e.g. banks recognising cryptocurrency's associated fiat currency value, notarisation that legally accepts blockchain timestamps as legitimate.

1) Peer-to-Peer Electricity Trade: Of the existing industry literature, a majority examines blockchain's role in enabling peer-to-peer $(\mathrm{P} 2 \mathrm{P})$ electricity trading on a local scale [37]. The P2P paradigm allows for the exchange of generated energy between involved parties, at least in a financial sense [51], [52]. No longer in a purely passive consumer role, these parties now act as prosumers (proactive consumers), who perform intelligent management and/or generation [24], [53]. This could disintermediate utilities.

$\mathrm{P} 2 \mathrm{P}$ trading schemes usually make use of energy tokenisation in some form. In data science, tokenisation is defined as the method of replacing sensitive, complex data with non-sensitive simple values, while storing actual values in a secure ledger [54]. Thus, in the case of energy tokenisation, a token is created for every unit of energy generated [42]. The value of a token will typically be attached to a specified energy value e.g. one token represents one $\mathrm{kW}$.h or $\mathrm{kW}$.min of electrical energy. Different to the data science definition of tokenisation above, the physical energy is not necessarily bound to a specific token, except in the case where storage of some kind can be implemented. Tokenisation allows energy to become tangible as a commodity, thus falling within the Sustaining Technology tier.

Suppose an individual or business, hereafter referred to as Prosumer A wishes to trade energy on a P2P network. A smart meter is installed at the premises. This device must have some means of communicating with the necessary blockchain, and would typically be IoT-enabled in some measure. A good example is that developed by Pylon Network [37], [55]. If the premises has a form of generating 
equipment (e.g. PV array, small wind generator), this might typically be required to be sub-metered. When Prosumer A generates, say, one kW.min of electricity from their home system for export, the meter registers this and an energy token Tokl is added to the blockchain under A's unique ownership ID (e.g. private key). Prosumer B, who wishes to purchase this energy, sends a unit of cryptocurrency (e.g. Bitcoin, Litecoin, or a stablecoin such as Tether) at a predetermined price. This price could be a fixed rate at an agreed tariff, or use some form of automated auctioning mechanism as in [56] or [57]. Tokl is transferred to Prosumer B's unique ID. When Prosumer B consumes this energy, their smart meter communicates with the blockchain, and the specific energy token is dissolved.

It is worth considering the Oracle problem [40]: the meter and underlying smart contract mechanism trust the input received to make decisions. Thus, if the meter is tampered with, it is theoretically possible to purchase or sell fraudulently. The blockchain is only as good as its input. Therefore some form of monitoring authority may be required to perform routine checks, in the same manner as electricity providers currently do. A number of the examples covered in this subsection combine tokenisation with smart contracts, thus tend towards the Evolutionary Technology tier classification.

In the P2P case, the decentralised nature of a blockchain-based environment allows for a trustfree energy market without a central mediator [20] (besides the trust put in the operation of the smart meter and the electrical installer). The dynamic nature of such a market allows for real-time distributed decisions [51], such as reacting to demand or fluctuating renewable generation. A P2P energy trading system has been successfully implemented on a microgrid level [58], [59]. Although energy exchange is usually limited to the geographical area, P2P energy trade can potentially help satisfy local energy demands. Transfer losses can be decreased in this manner [23], as local consumers can be given preference to encourage grid balancing.

The P2P concept is expanded further, with proposals for exchanges on an even smaller scale, in the form of machine-to-machine electricity trade [60], or between electric vehicles and the grid. A partial or total P2P ecosystem, as described above, would make extensive use of smart contracts for semiautonomous operation without human mediation, and thus fits within the Evolutionary Technology tier, although completely reliant on energy tokenisation as in the Sustaining Technology tier. Such an ecosystem is demonstrated visually in Figure 1. Most would require some form of smart metering with a means of communicating with the blockchain. P2P ecosystems may also employ smart contracts to implement derivatives, hedging and insurance mechanisms, with these smart contracts autonomously tracking assets, facilitating value transfer and distributing funds. This is similar to the workings of [43]. The energy industry is likely to undergo only minor changes at first, unless a pure $\mathrm{P} 2 \mathrm{P}$ is established. The latter scenario may take years to implement, if at all.

Large scale energy providers should investigate blockchain as platform for energy exchanges, so as to easily integrate into any future P2P-based platforms. The role of electricity wholesalers may be reduced by a significant measure, so energy providers could remove them as an intermediary by selling directly. Developers of lifestyle villages and housing complexes may be interested in implementing some form of P2P system. Alternatively, the tokenisation process potentially presents an accurate and secure metering method for grid operators and electricity wholesalers.

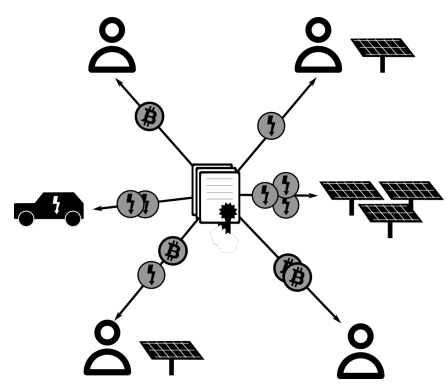

Fig. 1. Visual representation of a smart contract-based peer-to-peer energy trading environment.

2) Tokenised Energy Advanced Concepts: This section examines some of the possible advanced concepts and derivatives that are possible in a blockchain-based P2P tokenised electricity trading environment.

An advantage of the tokenised energy utilised by 
blockchain-based methods is the associated metadata. Metadata specific to the particular energy quantum is generated and included during the tokenisation process, and is potentially visible to all involved parties on the blockchain's distributed ledger. This attribute allows each token to include additional details of the unit of energy it represents, stored within the block of transactions, or as a second-layer metacoin [61]. The nature of generation (e.g. wind, solar, fossil fuel) can be attached to each token [62], as well as some form of geotag. If a consumer wishes to exclusively purchase energy produced from local or renewable sources they may do so, perhaps at a premium.

Thus, energy tokenisation allows guarantee of origin [62], which could underpin schemes whereby a consumers would pay a tariff based on their geographic grid distance from the producer. Likewise, the time of generation can be included for the specific token. This can be used to lock the unit of energy to a specific time-of-use period, which could discourage hoarding for use during peak periods, minimising the risk of grid imbalance. Furthermore, a demurrage mechanism, which diminishes the redemptive energy value of a token over time, is possible, as in [57]. Producers and consumers are thus incentivised to act fast, implicitly creating a time of use-style demand response mechanism. This tightens the coupling between physical energy use and the associated financial abstraction. This may allow for some form of electricity supply chain management.

In an unrestricted peer-to-peer energy trade environment, some parties could find themselves effectively independent from utility-scale generation and electricity wholesale companies. They would thus only pay some form of network access fee, perhaps a flat rate, to the network operator, and would have no enduring contractual relationship with any electricity supply company.

Notwithstanding the above, the fiat currency-based traditional energy sales model is likely to continue to serve as the average consumer's purchase method. Partial disruption is achieved at this level.

The methodology behind $\mathrm{P} 2 \mathrm{P}$ energy trading can be extended to Electric Vehicles $(E V s)$. The standard model for EV charging typically involves a home charging point or a paid public charging point. IoT- enabled vehicles are becoming an oft-discussed topic, especially in the case of bi-directional energy trade between EVs and/or the grid [63]. Blockchain has been suggested as a possible tool in this process, by facilitating advanced energy trade mechanisms [23], [64], [65], [66]. The methodology largely mirrors the P2P energy trading described above. EVs, when connected, can automatically and anonymously trade with nearby parties using smart contracts-based mechanisms, potentially easing grid congestion [66]. The vehicle may temporarily act as a local generator, if it is established to have excess energy stored. Furthermore, blockchain technology could enable shared EV charging from a single charging station, thus minimising charge port anxiety for users [67]. EV charging infrastructure designers may want to consider the above for projects in the near future, but are some time from any of these concepts being realised in a significant capacity. Those in the field of IoT-enabled vehicles could potentially benefit greatly from blockchain-based methods, however an adequate $\mathrm{P} 2 \mathrm{P}$ based ecosystem will need to be implemented first.

3) Grid-scale disruption: This tour of blockchain in electricity is now reaching the fringes of the Evolutionary Technology tier and is heading toward the Disruptive Technology tier, as attention shifts towards governance concepts.

A grid operator of some kind will always be required to install and maintain transmission and distribution infrastructure. This scenario is unlikely to be decentralised in any way, due to the skilled labour and informed planning required. However, the grid operator could be collaboratively owned. Parties could invest cryptocurrency into DAO to gain shares in the grid operator company. Stakeholders could earn a pro-rata share in the grid operating company's revenue as in the concepts mentioned in Section II-B2. The company would function along the lines of a non-profit organisation, with earnings being reinvested into grid maintenance and expansion. If automatic P2P trading becomes ubiquitous, commercial Megawatt-scale producers could sell exclusively on the blockchain, as mentioned before. Their role may be reduced, as distributed home-scale generation will have a higher level of penetration at this point. Furthermore, large scale generators may exist in 
some form of collaborative ownership, similar to the concept discussed in Section III-C2.

\section{Disruptive Technology}

Blockchain as a tool for governance and exotic ownership models is a fairly new idea, but is being investigated by a number of researchers and institutions. The general concept is that controlling decisions can be made via blockchain-based voting mechanisms [44] and could be extended to all involved parties. As before, a large amount of human involvement can be removed: what better manager/representative than a (theoretically) purely democratic and un-corruptable digital one [68]? A simple application is a secure and transparent voting mechanism [69]. Voting would be performed on the blockchain, making it secure and less vulnerable to tampering or voter fraud [70]. A public blockchain would also allow voting records to be completely transparent, but voters would retain their anonymity behind their unique public keys.

The next step involves automatically implementing and acting on policies. Management operations could largely be performed by smart contracts [69], in combination with the voting mechanisms above. Figure 2 shows two examples of digital ballots that may be used in the proposed system. Dues are automatically enforced with groups of interacting smart contracts [47]. It has even been argued that blockchain-based governance could be extended to the point of replacing the state altogether [71], but this is unlikely to see large-scale adoption soon, as it may be difficult to practically enforce obligations and act on breaches in contracts and dues. An interesting example within this tier is the Ethereumbased entity MakerDAO and its associated stablecoin DAI, which maintains a tight pegging to the US dollar using decentralised incentive structures. The core mechanism by which DAI maintains its pegging is by users locking assets into smart contract collateralised debt positions [43] to underpin the issuance of new DAI tokens. This mechanism, and other decentralised and anonymous governance structures, gives the DAI token a functional and stable monetary policy without any kind of central bank or other controlling entity.

A blockchain-based voting/governance system could make industries more democratic; representa-

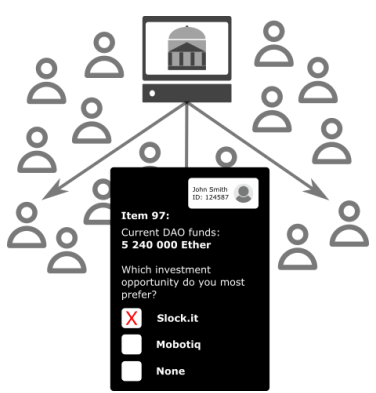

Fig. 2. Examples of digital ballots that may be used in blockchain governance.

tives would be elected directly by stakeholders as a part of a non-hierarchical model [69]. This method could benefit from advanced voting concepts, such as quadratic voting [72] or liquid democracy [73] (wherein individuals would have the option to elect specialist representatives within their various social circles).

1) Exotic Ownership Models: Once P2P energy trade has become more commonplace between prosumers, there are a number of interesting Disruptive Technology tier concepts, such as Virtual Power Plants, that can be implemented. Despite an increase in distributed generation capacity from prosumers, its impact on the grid as a whole is still relatively minor. This lack of effect may be due to a number of factors, such as difficulty in communication, volatility of potential export profits [24] and infrastructure cost, resulting in a high entry barrier for the general population. Prosumers can theoretically coordinate to act as a single aggregated service-centric entity. Retail suppliers are incentivised to provide higher prices for more a predictable supply [74]. This concept is known as a Virtual Power Plant (VPP) [75]. A smart contract may facilitate the consensus mechanism for a number of generating prosumers to form a VPP. Votes can be cast and, if unanimous, the smart contract arranges the members into a VPP and acts as the VPP mediator, liaising with the appropriate grid operator and fairly disbursing revenues. The mechanism can automatically decide which portions of multiple parties' generated energy to sell off at specific times so as to meet the obligations to the buying utility. It could also handle the allocation of proceeds from sales.

VPPs allow prosumers a certain level of "hedging". 
A specific VPP Member A's home generation could be under-performing at a certain time due to weather conditions, but Member B, who is based in another geographic location where weather conditions are favourable, may subsidise this lack of production. Similarly, Member A may contribute some energy production revenue to Member B when conditions are reversed. VPPs allow public participation in gridscale energy markets [76], thus serving as a more stable source of revenue for energy generation asset holders. Most existing proposed VPP strategies still involve some form of central mediator acting as the "power plant aggregator" [77], [75] [24]. The VPP-based ideas examined above consist of elements from all three tiers. VPPs and will certainly make use of energy tokenisation and likely some form of ownership ledger (Sustaining Technology tier), exist on some form of smart contract platform (as mentioned previously), which handles the exchanges of energy and currency (Evolutionary Technology tier) and will use some form of consensus mechanism for governance and decision making between stakeholders (Disruptive Technology tier). Generation leasing companies or companies with generation at a number of facilities may consider these concepts as a means of hedging revenue i.e. selling generation from multiple sources so as to guarantee a minimum daily production.

Continuing on the theme of collaborative generation, a blockchain-based energy purchase mechanism holds the potential to allow a shared large-scale economy of production. Generation assets can be installed in geographically ideal locations (e.g. solar arrays in sunnier climates), but profits are distributed amongst all stakeholders. This accomplishes financial securisation through blockchain [78]. Asset owners are then paid in cryptocurrency. If the market operator settles in cryptocurrency, these revenue shares are guaranteed. Alternatively, they can receive their share of energy tokens for self consumption. A similar leasing mechanism has been implemented on PV arrays in the case of [37], [79]. Forward and future contracts are used to hedge revenues for renewable generators.

2) Smart Contract Finance: Grid-scale renewable energy projects are notoriously capital intensive [80]. This high expense, coupled with the intermittent nature of renewable generation often makes finance difficult [42], [81]. Lenders may be unwilling to put forward the funds without a guarantee to make returns on investments. Many finance organisations are currently exploring smart contract technology as a potential hedging tool [9], and this may be extended to the renewable energy industry [82]. A smart contract may accept funds from investors, similar to a crowd funding process [25]. Once complete, the project proceeds may be shared pro-rata with investors [78], [83]. These results are all accomplished without any central mediator, with proceeds paid in cryptocurrency directly. The process roughly imitates the intended workings of Ethereum's DAO [9] by allowing public participation and ownership in large scale projects, while not depending on any single corporate entity. The secure public keys attached to each blockchain user makes authority management possible [25], adding a layer of security in this process. Thus, similar to how finance is approved in the traditional sense, a user's reliability can be assessed based on their payment and consumption history (both of which publicly available once their public key ID is identified), perhaps with a form of consensus voting mechanism allowing stakeholders to select partner investors.

Unlike other, more abstract, concepts in this tier, the idea of smart contract finance for large scale renewable energy projects has already seen some real-world manifestation [37], [83]. The idea does not necessarily depend on a the development of a P2P blockchain trading ecosystem. Proceeds are simple to divide (especially with market operator co-operation), even if fiat currency is used. Thus, it should be a consideration for companies attempting to secure funding for such projects.

In a similar vein, but on a smaller scale, generation assets for homes and businesses can be purchased on credit and paid off over time. The smart meter can intervene and disconnect supply if payments are not honoured. This system is comparable to the workings of M-PAYG [37], who provide "pay as you go" solar PV leasing. If energy is exported for profit, some portion can automatically be put towards payments on the equipment itself. This could be based on a smart contract, in combination with a $\mathrm{P} 2 \mathrm{P}$ electricity 
trading platform.

\section{Conclusions}

Owing to excitement around the subject, blockchain has been subject to much attention. Research into its usage has been extensive, with a variety of ideas ranging from plausible to more exotic. The electrical energy field is not exempt, and has seen its fair share of research and business implementations. As such, engineering and management personnel may find themselves confused as to blockchain's true usefulness and likely impact in their specific sectors. This study has elucidated a tiered taxonomy of industry disruption by blockchain technology. This taxonomy is used as a framework to guide a literature review on blockchain's usage in the electrical energy industry. The likeliness of adoption of the various concepts are discussed. Although there is certainly potential for significant change in the industry, there are many barriers to implementation (especially for more exotic concepts) and the actual depth of disruption is yet to be seen.

Ideas grouped in the first tier seem most likely to occur. One recommendation is that electricity wholesalers should most likely invest in developing cryptocurrency-based payment options, as this change would be relatively simple to implement and could largely act as future-proofing in terms of advanced payment layers. Cryptocurrency as a payment layer has seen some mainstream acceptance, and this trend is likely to continue. Those involved in generation asset management or carbon trade should consider a novel blockchain as secure and transparent ledger, which would make adapting to large-scale roll outs of distributed ledgers easier in the future.

The second tier seems less likely to occur in the near future. That said, peer-to-peer trading mechanisms have already been implemented on a small scale, and those involved in generation leasing could consider this mechanism. IoT-enabled smart metering developers could allow for some form of blockchain interfacing in future products. Developers of housing complexes could consider a peer-to-peer trading system between units. If this level of disruption is achieved, energy wholesalers are most at risk, and should investigate expanding or pivoting into new services. For both this tier and the first tier it is important to recall the Oracle problem, which always requires a degree of trust in some informationproviding entity for any smart contract which requires off-chain data.

In terms of the third tier, very high-level concepts are present, and are least likely to see implementation in the near future, if at all. Regulation changes and resistance of existing entities may prove too strong as barriers of entry. While the technology is promising as a radical tool for reimagining management and governance, in the authors' opinion those in engineering management do not need to worry about losing their jobs to smart contracts any time soon.

\section{INTEREST Disclosure}

The authors hold cryptographic assets. 
TABLE II. Summary of Blockchain Energy Industry Disruption Classification

\begin{tabular}{lll} 
& Defining Applications & \multicolumn{1}{c}{ Core Concepts } \\
\hline Sustaining Technology & Commodities, Trading. & Cryptocurrency payment layer. \\
& Digital notarisation for ownership and \\
carbon credits tracking.
\end{tabular}
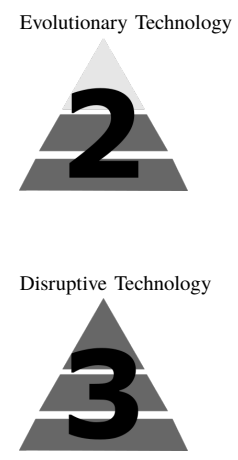

\begin{abstract}
Derivatives,
Ecosystems.
\end{abstract}

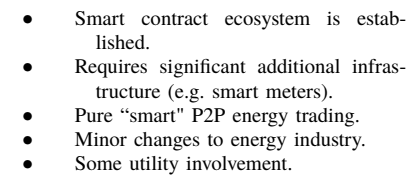

Structure, Organisation,
Governance, Disintermediation.
- Blockchain-based decentralised regulation.

- Total overhaul of payment and governance structure.

- Largely replaces major utilities and energy wholesalers.

- Minimised role for grid operators.

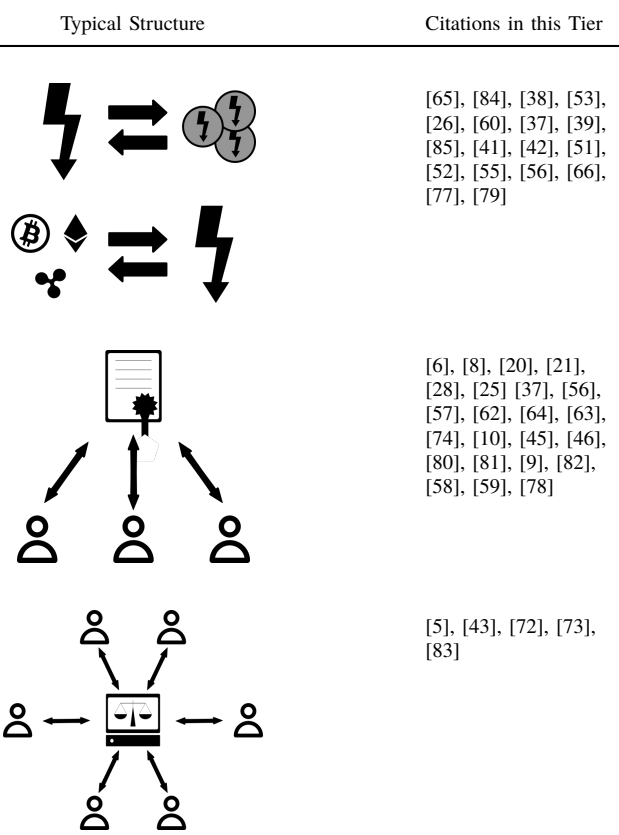

\section{REFERENCES}

[1] S. Nakamoto, "Bitcoin: A peer-to-peer electronic cash system," 2008.

[2] T. T. A. Dinh, R. Liu, M. Zhang, G. Chen, B. C. Ooi, and J. Wang, "Untangling blockchain: A data processing view of blockchain systems," IEEE Transactions on Knowledge and Data Engineering, vol. 30, no. 7, pp. 1366-1385, 2018.

[3] K. Chuen, D. Guo, and Y. Wang, "Cryptocurrency: A new investment opportunity?" The Journal of Alternative Investments, vol. 20, no. 3, pp. 1520-3255, 2017.

[4] E. Deirmentzoglou, P. Georgios, and C. Patsakis, "A survey on long-range attacks for proof of stake protocols," IEEE Access, vol. 7, pp. 28712-28725, 2019.

[5] N. Szabo, "Formalizing and securing relationships on public networks," First Monday, vol. 2, no. 9, 1997.

[6] L. Thomas, C. Long, P. Burnap, J. Wu, and N. Jenkins, "Automation of the supplier role in the gb power system using blockchain-based smart contracts," CIRED Open Access Proceedings Journal, vol. 2017, no. 1, pp. 26192623, 2017.

[7] B. Smith, What are the three generations of blockchain, and how are they similar to the web? 2018. [Online]. Available: https : / / www . coininsider . com / three generations-of-blockchain/.

[8] G. Wood, "Ethereum: A secure decentralised generalised transaction ledger," Ethereum Project Yellow Paper, 2014.
[9] I. Eyal, "Blockchain technology: Transforming libertarian cryptocurrency dreams to finance and banking realities," Computer, vol. 50, no. 9, pp. 38-49, 2017.

[10] M. J. M. Chowdhury, A. Colman, M. A. Kabir, J. Han, and P. Sarda, "Blockchain as a notarization service for data sharing with personal data store," in 12th IEEE International Conference On Big Data Science And Engineering, IEEE, 2018.

[11] (). State of the dapps, [Online]. Available: https://www. stateofthedapps.com/.

[12] M. E. Peck, "Do you need a blockchain?" IEEE Spectrum, vol. 54, no. 10, pp. 36-60, 2017.

[13] J. Al-Jaroodi and N. Mohamed, "Blockchain in industries: A survey," IEEE Access, vol. 57, pp. 3650036 515, 2019.

[14] S. M. Gainsbury and A. Blaszczynski, "How blockchain and cryptocurrency technology could revolutionize online gambling," Gaming Law Review, vol. 21, no. 7, pp. 482-492, 2017.

[15] V. Gatteschi, F. Lamberti, C. Demartini, C. Pranteda, and V. Santamaria, "Blockchain or not blockchain, that is the question of the insurance and other sectors," IT Professional, vol. 99, pp. 1-1, 2017.

[16] M. Nofer, P. Gomber, O. Hinz, and D. Schiereck, "Blockchain," Business and Information Systems Engineering, vol. 59, no. 3, pp. 183-187, 2017.

[17] J. Veuger, "Trust in a viable real estate economy with disruption and blockchain," Facilities, vol. 36, no. 1/2, pp. 103-120, 2017.

[18] Q. Zhu and M. Kouhizadeh, "Blockchain technology, supply chain information, and strategic product deletion 
management," IEEE Engineering Management Review, vol. 47, no. 1, pp. 36-44, 2019.

[19] A. Savelyev, "Copyright in the blockchain era: Promises and challenges," Computer Law and Security Review, vol. 34, no. 3, pp. 550-561, 2018.

[20] E. Mengelkamp, B. Notheisen, C. Beer, D. Dauer, and C. Weinhardt, "A blockchain-based smart grid: Towards sustainable local energy markets," Computer Science Research and Development, vol. 33, no. 1, pp. 207-214, Feb. 2018.

[21] M. Mihaylov, S. Jurado, K. Van Moffaert, N. Avellana, and A. Nowé, "NRG-X-Change-a novel mechanism for trading of renewable energy in smart grids.," in SMARTGREENS, 2014, pp. 101-106.

[22] J. Aron, "What's wrong with Bitcoin?" New Scientist, vol. 221, no. 2955, pp. 19-20, 2014.

[23] Z. Li, J. Kang, R. Yu, D. Ye, Q. Deng, and Z. Yan, "Consortium blockchain for secure energy trading in industrial internet of things," IEEE Transactions on Industrial Informatics, vol. 14, no. 8, pp. 3690-3700, 2018.

[24] T. Morstyn, N. Farrell, and M. D. Darby Sarah J McCulloch, "Using peer-to-peer energy-trading platforms to incentivize prosumers to form federated power plants," Nature Energy, vol. 4, pp. 94-101, 2018.

[25] Y. Huang, P. Yang, Y. Liu, and Y. Chen, "A design of photovoltaic plants financing platform based on blockchain technology," in International Conference on Power System Technology (POWERCON), IEEE, 2017, pp. 4251-4256.

[26] K. P. Sharma, M.-Y. Chen, and H. Park, "A software defined fog node based distributed blockchain cloud architecture for iot," IEEE Access, vol. 6, pp. 115-124, 2017.

[27] L. Thomas, L. Chao, P. Burnap, J. Wu, and N. Jenkins, "Automation of the supplier role in the GB power system using blockchain based smart contracts," in CIRED, Glasgow, Scotland, May 2017.

[28] L. Wu, K. Meng, S. Xu, S. Li, M. Ding, and Y. Suo, "Democratic centralism: A hybrid blockchain architecture and its applications in energy internet," in International Conference on Energy Internet Conference Proceedings, IEEE, 2017, pp. 176-181.

[29] E. Rogers, Diffusion of innovations. Free Press, 1962.

[30] S. Sinofsky. (2014). The four stages of disruption, [Online]. Available: https://a16z.com/2014/01/14/thefour-stages-of-disruption/.

[31] E. Küblerr-Ross', On death and dying. The Macmillan Company, 1969.

[32] R. Adler, "Navigating continual disruption," 2015. [Online]. Available: http://www.johnseelybrown.com/ navigatingdistruption.pdf.

[33] K. Kilkki, M. Mäntylä, K. Karhu, and H. Hämmäinen Heikki adn Ailisto, "A disruption framework,"
Technological Forecasting and Social Change, vol. 129, pp. 275-284, 2018.

[34] S. Wang, L. Ouyang, Y. Yuan, X. Ni Xiaochun Han, and F.-Y. Wand, "Blockchain-enabled smart contracts: Architecture, applications, and future trends," IEEE Transactions on Systems, Man, and Cybernetics: Systems, pp. 1-12, 2019.

[35] L. Hongfang, K. Huang, M. Azimi, and L. Guo, "Blockchain technology in the oil and gas industry: A review of applications, opportunities, challenges, and risks," IEEE Access, vol. 7, pp. 41 426-41 444, 2019.

[36] N. Szabo, Quote, 2018. [Online]. Available: https:// twitter.com/nickszabo4/status/1009870550741671936.

[37] M. Andoni, V. Robu, D. Flynn, S. Abram, D. Geach, D. Jenkins, P. McCallum, and A. Peacock, "Blockchain technology in the energy sector: A systematic review of challenges and opportunities," Renewable and Sustainable Eneregy Reviews, vol. 100, pp. 143-174, 2019.

[38] Y. Yuan and F.-Y. Wang, "Blockchain and cryptocurrencies: Model, techniques, and applications," IEEE Transactions on Systems, Man, and Cybernetics: Systems, vol. 48, no. 9, pp. 1421-1428, 2019.

[39] J. C. Mendoza-Tello, H. Mora, F. Pujol-López, and M. D. Lytras, "Social commerce as driver to enhance trust and intention to use cryptocurrencies for electronic payments," IEEE Access, vol. 6, pp. 50737-50751, 2018.

[40] E. T. Barr, M. Harman, P. McMinn, M. Shahbaz, and S. Yoo, "The oracle problem in software testing: A survey," IEEE Transactions on Software Engineering, vol. 41, no. 5, pp. 507-525, 2015.

[41] G. Prisco, "Bankymoon introduces Bitcoin payments to smart meters for power grids," Bitcoin Magazine, Apr. 2015. [Online]. Available: https://bitcoinmagazine. com/articles/bankymoon-introduces-bitcoin-paymentssmart-meters-power-grids-1429824186/.

[42] J. Dispenza, C. Garcia, and R. Molecke, "Energy efficiency coin (eecoin) a blockchain asset class pegged to renewable energy markets," 2017.

[43] R. Christensen, N. Mushegian, D. Brockman, K. Rowe, A. Milenius, and R. Zurrer, "The Dai stablecoin system," 2018.

[44] P. Boucher, "How blockchain technology could change our lives," 2017.

[45] M. del Castillo, NASDAQ explores how blockchain could fuel solar energy market, 2016. [Online]. Available: https://www.coindesk.com/nasdaq-blockchainsolar-power-market.

[46] M. J. Ashley and M. S. Johnson, "Establishing a secure, transparent, and autonomous blockchain of custody for renewable energy credits and carbon credits," IEEE Engineering Management Review, vol. 48, no. 4, pp. 100-102, 2018. 
[47] A. Norta, "Designing a smart-contract application layer for transacting decentralized autonomous organizations," in Smart Blockchain 2018 Proceedings, Springer, Cham, vol. 1, 2018, pp. 595-604.

[48] V. Gatteschi, F. Lamberti, C. Demartini, C. Pranteda, and V. Santamaria, "Blockchain and smart contracts for insurance: Is the technology mature enough?" Future Internet, vol. 10, no. 20, pp. 550-561, 2018.

[49] W. Wang, D. T. Hoang, P. Hu, Z. Xiong, D. Niyato, P. Wang, Y. Wen, and D. I. Kim, "A survey on consensus mechanisms and mining strategy management in blockchain networks," IEEE Access, vol. 7, pp. 22 328-22 370, 2018.

[50] K. O'Hara, "Smart contracts-dumb idea," IEEE Internet Computing, vol. 21, pp. 97-101, 2017.

[51] M. L. D. Silvestre, P. Gallo, M. G. Ippolito, E. R. Sanseverino, and G. Zizzo, "A technical approach to the energy blockchain in microgrids," IEEE Transactions on Industrial Informatics, vol. 14, no. 11, pp. 4792-4803, 2018.

[52] C. Zhang, J. Wu, C. Long, and M. Cheng, "Review of existing peer-to-peer energy trading projects," in International Conference on Applied Energy, Elsevier, 2017, pp. 2563-2568.

[53] A. Dimeas, S. Drenkard, Hatziargyriou, S. Karnouskos, K. Kok, and J. Ringelstein, "Smart houses in the smart grid: Developing an interactive network.," IEEE Electrification Magazine, vol. 2, no. 1, pp. 81-93, 2014.

[54] R. C. Goldstein and C. Wagner, "Database management with sequence trees and tokens," IEEE TRANSACTIONS ON KNOWLEDGE AND DATA ENGINEERING, vol. 9, no. 1, pp. 186-192, 1997.

[55] P. Network, Pylon network, 2019. [Online]. Available: https://pylon-network.org/.

[56] W. Zhong, K. Xie, Y. Liu, C. Yang, and S. Xie, "Auction mechanisms for energy trading in multi-energy systems," IEEE Transactions on Industrial Informatics, vol. 14, no. 4, pp. 1511-1521, 2018.

[57] M. T. Devine and P. Cuffe, "Blockchain electricity trading under demurrage," IEEE Transactions on Smart Grid, vol. 10, no. 2, pp. 2323-2325, 2019.

[58] R. Sharma, "Brooklyn microgrid brings community microgrid to New York," EnergyCentral, Dec. 2016.

[59] M. E. Peck and D. Wagman, "Do you need a blockchain?" IEEE Spectrum, vol. 54, no. 10, pp. 56-61, 2017.

[60] J. J. Sikorski, J. Haughton, and M. Kraft, "Blockchain technology in the chemical industry: Machine-tomachine electricity market," Applied Energy, vol. 195, pp. 234-246, 2017.

[61] P. Tasca, A. Tomaso, and T. Di Matteo, "Blockchain technologies: The foreseeable impact on society and industry," IEEE Computer, vol. 50, no. 9, pp. 18-28, 2017.
[62] T. Morstyn and M. McCulloch, "Multi-class energy management for peer-to-peer energy trading driven by prosumer preferences," IEEE Transactions on Power Systems, 2018.

[63] F. Berthold, A. Ravey, B. Blunier, D. Bouquain, S. Williamson, and A. Miraoui, "Design and development of a smart control strategy for plug-in hybrid vehicles including vehicle-to-home functionality," IEEE Transactions on Transportation Electrification, vol. 1, no. 2, pp. 168-177, 2015.

[64] X. Huang, C. Xu, P. Wang, and H. Liu, "Lnsc: A security model for electric vehicle and charging pile management based on blockchain ecosystem," IEEE Access, vol. 6, pp. 13 565-13 574, 2018.

[65] V. Sharma, "An energy-efficient transaction model for the blockchain-enabled internet of vehicles (iov)," IEEE Communications Letters, vol. 23, no. 2, pp. 246-249, 1997.

[66] J. Kang, R. Yu, X. Huang, S. Maharjan, Y. Zhang, and E. Hossain, "Enabling localized peer-to-peer electricity trading among plug-in hybrid electric vehicles using consortium blockchains," IEEE Transactions on Industrial Informatics, vol. 13, no. 6, pp. 3154-3164, 2017.

[67] P. Ferraro, C. King, and R. Shorten, "Distributed ledger technology for smart cities, the sharing economy, and social compliance," IEEE Access, vol. 6, pp. 62 72862746, 2018.

[68] N. Kshetri, "Will blockchain emerge as a tool to break the poverty chain in the global south?" Third World Quaterly, vol. 38, no. 8, pp. 1710-1732, 2017.

[69] O. Svein, J. Ulbacht, and M. Janssen, "Blockchain in government: Benefits and implications of distributed ledger technology for information sharing," Government Information Quarterly, vol. 34, no. 3, pp. 355-364, 2017.

[70] Y. Cai and D. Zhu, "Fraud detections for online businesses: A perspective from blockchain technology," Financial Innovation, vol. 2, no. 20, 2016.

[71] M. Atzori. (2016). Blockchain technology and decentralized governance: Is the state still necessary? [Online]. Available: https://papers.ssrn.com/sol3/papers.cfm? abstract_id=2709713.

[72] E. G. Weyl and E. A. Posner, Radical Markets. Princeton University Press, 2018.

[73] S. Siri, V. Deville, P. Berman, E. Medina, H. Stephens, S. Miller, A. Bacelar, D. Wilson, M. Williams, L. Margot-Duclot, F. Alvarez, C. Grau, P. Schurman, A. J. Benson, G. Stupenengo, and L. Isasmendi, "The social smart contract," 2018.

[74] L. Han, T. Morstyn, and M. McCulloch, "Constructing prosumer coalitions for energy cost savings using cooperative game theory," in 2018 Power Systems Computation Conference (PSCC), IEEE, 2018. 
[75] D. Koraki and K. Strunz, "Wind and solar power integration in electricity markets and distribution networks through service-centric virtual power plants," IEEE Transactions on Power Systems, vol. 33, no. 1, pp. 473-485, 2018.

[76] S. Wassermann, M. Reeg, and K. Niehaus, "Current challenges of Germany's energy transition and competing strategies of challenger and incumbents: The case of direct marketing of electricity from renewable energy sources," Energy Policy, vol. 76, pp. 66-75, 2015.

[77] P. Siano, G. De Marco, A. Rolán, and V. Loia, "A survey and evaluation of the potentials of distributed ledger technology for peer-to-peer transactive energy exchanges in local energy markets," IEEE Systems Journal, pp. 1-13, 2019.

[78] E. Foley, "The investigation of blockchain technology as a disruptive financing instrument for renewable energy," Master's thesis, University College Dublin, 2019.

[79] A. Cambridge, How does sun exchange work? 2018. [Online]. Available: https://www.youtube.com/watch? $\mathrm{v}=\mathrm{dWifCCibhng}$.

[80] D. X. Yang and P. Y. Nie, "Influence of optimal government subsidies for renewable energy enterprises," IET Renewable Power Generation, vol. 10, no. 9, pp. 1413-1421, 2016.

[81] J. Dispenza, Enledger, 2019. [Online]. Available: https: //www.enledger.io/.

[82] D. Bates, Impactppa bringing blockchain to renewable energy finance, 2017. [Online]. Available: https://www. impactppa . com/uncategorized/impactppa - bringing blockchain-to-renewable-energy-finance- $2 /$.

[83] D. Solodukha, O. Solodukha, R. Weglarek, M. Seidel, A. Melekhavets, K. Thiessen, D. Degtyarev, A. Shcherbina, A. Medvedev, L. Kreinin, M. Zaks, and E. Arkhipov, "Solar dao," 2017.

[84] M. Swan, Blockchain: Blueprint for a new economy. "O'Reilly Media, Inc.", 2015.

[85] C. Marckx, Dutch energy supplier bas to accept Bitcoin, 2014. [Online]. Available: https://www.ccn.com/dutchenergy-supplier-bas-to-accept-bitcoin. 